\title{
Streptococcus agalactiae (Group B Streptococcus) - An Uncommon Cause of Postpartum Spinal Epidural Abscess
}

\author{
Adiel Cohen 1* (D), Elchanan Parnasa1* (D) Nurith Hiller², Oren Wasser ${ }^{1}$ (D) , Josh E Schroeder ${ }^{3}$, \\ Hananel Shear-Yashuv ${ }^{3}$, Jacob Strahilevitz ${ }^{4}$ (D) \\ 1 Hadassah-Hebrew University Medical School, Jerusalem, Israel. \\ 2 Department of Radiology, Hadassah-Hebrew University Medical Center, Mount Scopus Campus, Jerusalem, Israel. \\ 3 Department of Orthopedic Surgery, Hadassah-Hebrew University Medical Center, Jerusalem, Israel. \\ 4 Department of Clinical Microbiology and Infectious Diseases, Hadassah-Hebrew University Medical Center, Jerusalem, Israel. \\ * Both authors contributed equally to this manuscript.
}

\section{ABSTRACT}

A nulliparous previously healthy pregnant woman who suffered from postpartum spinal epidural abscess due to Streptococcus agalactiae which probably evolved during delivery.

Keywords: Streptococcus agalactiae, spinal epidural abscess, postpartum

\section{INTRODUCTION} S pinal epidural abscess is a medical emergency because of the possible development of severe complications, including irreversible paralysis. The most common infecting pathogen is Staphylococcus aureus (1). Streptococcus agalactiae (Group B haemolytic streptococcus, GBS) has infrequently been described as the causative pathogen in cases of spinal epidural abscess.

We describe a woman who suffered from a postpartum spinal epidural abscess because of Streptococcus agalactiae (GBS), which probably evolved during delivery.

To our knowledge, the last reported case of GBS epidural abscess in a postpartum woman was reported in 1997 by Jenkin G et al. (2). We report an additional case of this uncommon condition.

\section{Case Presentation}

A 29-year-old nulliparous healthy pregnant woman began suffering from fluctuating low-back pain since the fourth month of her pregnancy. She delivered uneventfully at gestational week via vaginal birth, without the use of epidural anaesthesia.

Corresponding Author: Jacob Strahilevitz

E-mail: jstrahilevitz@hadassah.org.il

Received: February 5, 2021 Accepted: March 29, 2021 Published: April 30, 2021

\section{Suggested citation:} Cohen A, Parnasa E, Hiller N, Wasser O, Schroeder JE, Shear-Yashuv H, et al. Streptococcus agalactiae (Group B Streptococcus) - An Uncommon Cause of Postpartum Spinal Epidural Abscess. Infect Dis Clin Microbiol 2021; 1: 31-34.

DOI: 10.36519/idem.2021.24 

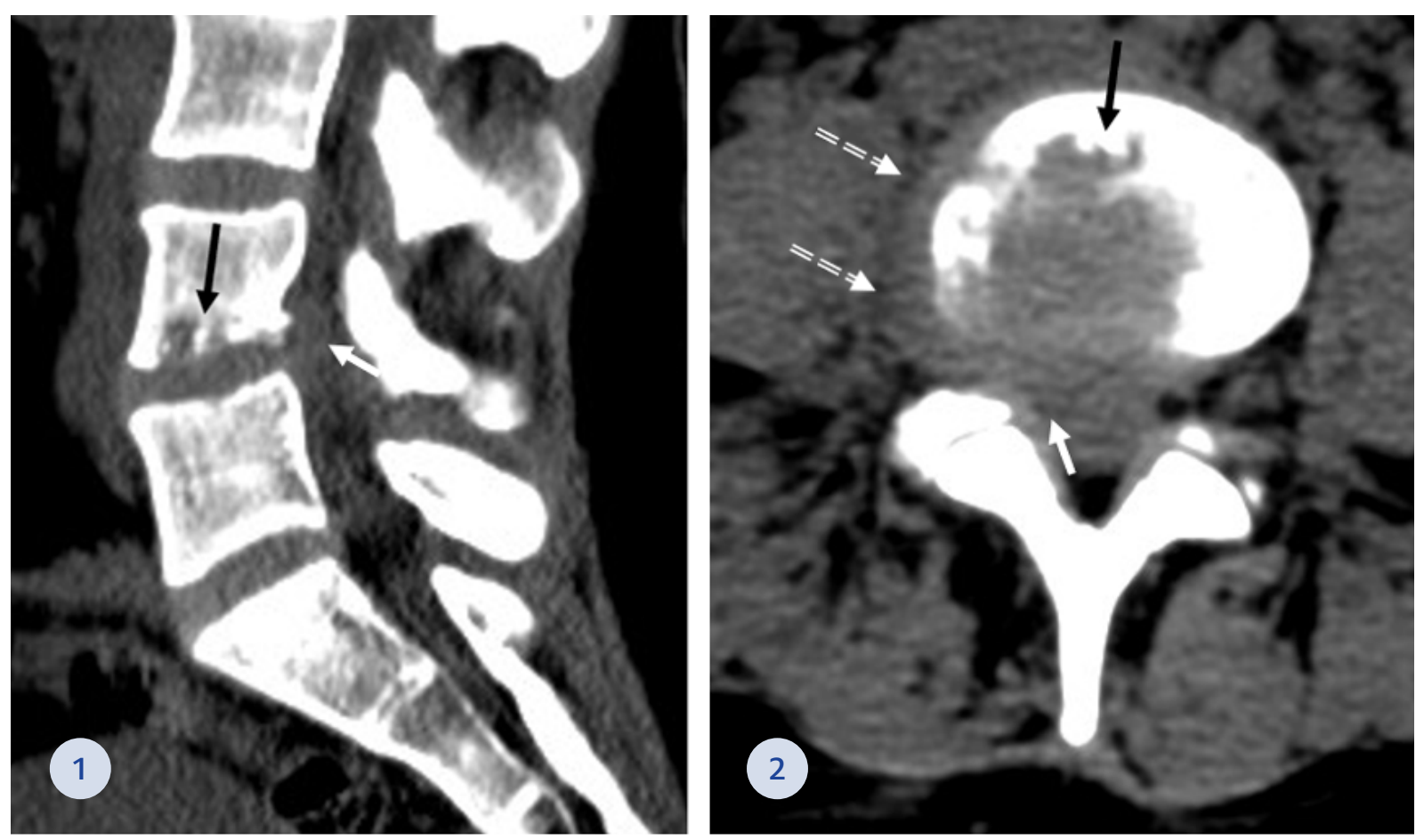

Figure 1-2. CT of the lumbar spine at the sagittal (1) and axial (2) planes showing L4-L5 right paracentral epidural (white arrows) and right paravertebral (dashed arrows) mass. Marked irregularity of the lower L4 endplate was also seen (black arrows).

In her urine screening culture performed several months before delivery grew GBS. She, however, declined antibiotic treatment.

Three weeks after the delivery, she presented to the emergency department (ED), complaining of low back pain radiating to the right leg. She had neither fever nor motor weakness, sensory disturbance or sphincters incontinence.

On presentation, physical examination for lower back tenderness was significant. A complete neurological exam was unnecessary. A Computed Tomography (CT) scan of the lumbosacral spine (Figure 1-2) was notable for suspected disc space infection at the level of L4-L5 with right foraminal and lateral extension compressing the thecal sac and the right L4 nerve root. Paracentral epidural soft tissue swelling was demonstrated compressing the right L5 nerve root. A further investigation of Magnetic Resonance Imaging (MRI) of the lumbosacral spine (Figure 3-4) showed bone marrow oedema and hyperemia at the L4-L5 level with posterior epidural inflammatory mass ( $3 \mathrm{~mm}$ diameter) narrowing the spinal canal and markedly compressing the thecal sac. The white blood cell count was 9.7 109/L (normal range: 4.05-11.84 109/L), C-Reactive Protein increased from $10 \mathrm{mg} / \mathrm{dL}$ at her last ED visit to 18 $\mathrm{mg} / \mathrm{dL}$ (normal range: 0-0.5 mg/dL) at her hospitalization, and the erythrocyte sedimentation rate was $50 \mathrm{~mm} / \mathrm{hr}$ (normal range: $1-20 \mathrm{~mm} / \mathrm{hr}$ ). In five sets of blood cultures obtained during her hospitalization grew GBS.

The patient underwent an emergency right L4-L5 hemilaminectomy. A non-suppurative inflammatory process was identified. Cultures from the inflammatory process were also positive for GBS.

The postoperative course was uneventful. The patient was treated with intravenous ceftriaxone and continued treatment with continuous Penicillin G, 20 million units daily for six weeks via a peripherally inserted central catheter (PICC line). Complete recovery of symptoms was observed at a follow-up visit three months later. 

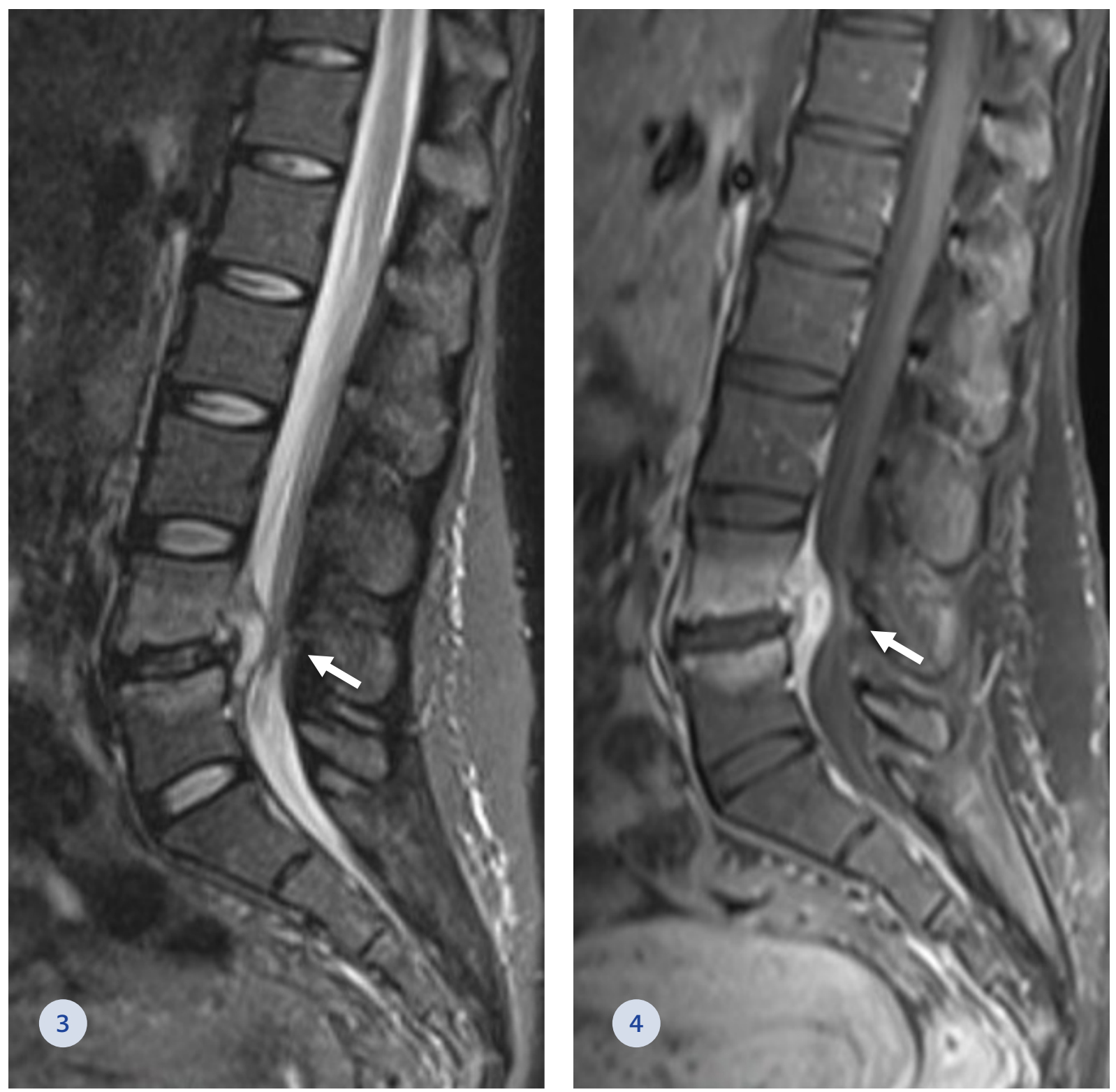

Figure 3-4. Magnetic Resonance Imaging of the lumbar spine in short tau inversion recovery (STIR) (3) and post gadolinium T1 fat-suppressed (4) sagittal images. Bone marrow oedema and hyperemia are demonstrated (white arrows) at the L4-L5 level, with posterior epidural inflammatory mass narrowing the spinal canal and markedly compressing the thecal sac.

\section{DISCUSSION}

Spinal epidural abscess is a rare but serious condition causing significant morbidity and mortality. It usually progresses from backache and focal vertebral pain, with tenderness on percussion to nerve root pain, manifesting with radiculopathy, paresthesia, or both, followed by spinal cord dysfunction, characterized by defects of motor, sensory, or sphincter function and paraplegia. The diagnosis, however, may be easily missed because the classic triad of back pain, fever, and neurologic deficits is seen in the minority of patients (3). Most cases of hematogenous dissemination to the spine from foci elsewhere in the body have been associated with an identifiable risk factor, mainly diabetes mellitus, obesity, chronic steroid use, chronic kidney disease, malignancy and alcoholism. (4). Staphylococcus aureus is the most common causal pathogen in cases of spinal epidural abscess, whereas GBS is a rare 
cause of vertebral osteomyelitis and spinal epidural abscess (1,5-7). GBS is a common colonizer of the genital area or lower gastrointestinal tract. The main concern of such carriage is a maternal-to-infant transmission that occurs in utero by the ascending route or delivery time. Nevertheless, as our patient demonstrated, pregnant women who are carriers of GBS might also be at an increased risk of spread from the genitourinary system to the spine. Normal bone is highly resistant to infection. The seeding of the bacteria into the vertebra was most likely caused by trauma during the child delivery. As the baby passes through the canal, micro and macro trauma may lead to haematogenous systemic spread leading to the spinal canal. This concern is particularly noteworthy because GBS infection incidence in adults has increased in the past few years (8). It is important for the obstetric community to be aware that treating the GBS antepartum may prevent such seeding.

\section{CONCLUSION}

Our case demonstrates the importance of maintaining a high index of suspicion for disc space infection in case of peripartum acute back pain, particularly in women with known urogenital colonization by a potential pathogen.
Peer-review: Externally peer-reviewed

Author Contributions: Concept - A.C., E.P., J.S.; Design - A.C., E.P., J.S.; Supervision - A.C., E.P., J.S.; Data Collection and/or Processing - A.C., E.P., J.S.; Analysis and/or Interpretation - A.C., E.P., J.S.; Literature Review - A.C., E.P., J.S.; Writer - A.C., E.P., N.H., J.S.; Critical Reviews - A.C., E.P., N.H., O.W., J.E.S, H.S.Y., J.S.
Conflict of Interest: The authors have no conflict of interest to declare.

Financial Disclosure: The authors declared that this study has received no financial support.

\section{REFERENCES}

1 Vakili M, Crum-Cianflone NF. Spinal epidural abscess: a series of 101 cases. Am J Med 2017; 130: 1452-63.

2 Jenkin G, Woolley IJ, Brown GV, Richards MJ. Postpartum epidural abscess due to group B Streptococcus. Clin Infect Dis 1997; 25: 1249. https://doi.org/10.1086/516961.

3 Bond A, Manian FA. Spinal epidural abscess: a review with special emphasis on earlier diagnosis. Biomed Res Int 2016; 2016 : 1614328. https://doi.org/10.1155/2016/1614328.

4 Bureta C, Tominaga H, Yamamoto T, Setoguchi T, Kawamura H, Nagano S, et al. Lumbar spine epidural abscess and facet joint septic arthritis due to Streptococcus agalactiae: a case report. BMC Surg 2018; 18: 16. https://doi.org/10.1186/s12893$\underline{018-0350-2 .}$
5 Kernéis S, Plainvert C, Barnier JP, Tazi A, Dmytruk N, Gislain $B$, et al. Clinical and microbiological features associated with group B Streptococcus bone and joint infections, France 20042014. Eur J Clin Microbiol Infect Dis 2017; 36: 1679-84.

6 Smith EM, Khan MA, Reingold A, Watt JP. Group B streptococcus infections of soft tissue and bone in California adults, 1995-2012. Epidemiol Infect 2015; 143: 3343-50

7 Solís-Garcia del Pozo J, Martinez-Alfaro E, Abad L, Solera J. Vertebral osteomyelitis caused by Streptococcus agalactiae. J Infect 2000; 41: 84-90.

8 Raabe VN, Shane AL. Group B Streptococcus (Streptococcus agalactiae). Microbiol Spectr 2019; 7: 10. 